\title{
Hospital outcome of concomitant tricuspid annuloplasty during totally endoscopic mitral valve surgery: a propensity matched study
}

\author{
Ling-Chen Huang ${ }^{1,2}$, Qi-Chen $\mathrm{Xu}^{1}$, Dao-Zhong Chen ${ }^{1}$, Xiao-Fu Dai ${ }^{1}$, Liang-Wan Chen ${ }^{1,2}$ \\ ${ }^{1}$ Department of Cardiovascular Surgery, Union Hospital, Fujian Medical University, Fuzhou, China; ${ }^{2}$ Fujian Key Laboratory of Cardio-thoracic \\ Surgery, Fujian Medical University, Fuzhou, China \\ Contributions: (I) Conception and design: LW Chen, LC Huang; (II) Administrative support: LW Chen; (III) Provision of study materials or patients: \\ DZ Chen, XF Dai; (IV) Collection and assembly of data: LC Huang, QC Xu; (V) Data analysis and interpretation: LC Huang, QC Xu; (VI) \\ Manuscript writing: All authors; (VII) Final approval of manuscript: All authors. \\ Correspondence to: Liang-Wan Chen. Department of Cardiovascular Surgery, Union Hospital, Fujian Medical University, Fuzhou 350001, China. \\ Email: gzlde0323@msn.com.
}

Background: This study aimed to determine how concomitant tricuspid annuloplasty (TAP) affects the clinical outcomes of patients undergoing totally endoscopic mitral valve surgery.

Methods: This was a single-centre, retrospective study. Between January 2019 and June 2020, 143 patients who underwent totally endoscopic mitral valve surgery in our institution were enrolled. Ninety-two patients who underwent isolated mitral valve surgery were categorized into the minimally invasive mitral valve surgery (MIMVS) group ( $n=92)$, and patients who underwent mitral valve surgery with concomitant TAP were categorized into the MIMVS-TAP ( $\mathrm{n}=51)$ group. Clinical data were collected from all patients, including demographic and perioperative data. We conducted propensity score matching (PSM) by using one-to-one ratio nearest-neighbour matching for baseline demographic data and tricuspid valve-related parameters. Forty patients in each group were matched in this way. Parametric and nonparametric tests were performed for data analysis.

Results: Statistically, postoperative mortality within 30 days was not significantly different between the two groups $(\mathrm{P}=1)$. No differences were found in serious adverse events, such as stroke or third-degree conduction block, between the two groups after 1:1 PSM $(\mathrm{P}=1$ and $\mathrm{P}=0.480$, respectively). The mean operation time for the MIMVS+TAP group was longer $(232.13 \pm 36.05 \mathrm{~min})$ than that for the MIMVS group $(204.25 \pm 28.49 \mathrm{~min}$; $\mathrm{P}<0.001)$. The same was true for the cardiopulmonary bypass $(\mathrm{CPB})$ time $(169.48 \pm 25.96$ vs. $153.10 \pm 23.00 \mathrm{~min}$; $\mathrm{P}=0.004)$ and aortic clamp time $(110.80 \pm 17.37$ vs. $101.00 \pm 14.38 \mathrm{~min} ; \mathrm{P}=0.005)$. The duration of the intensive care unit stay and the overall postoperative length of stay were not different between the two groups $(\mathrm{P}=0.734$ and $\mathrm{P}=0.472$, respectively). The postoperative systolic pulmonary artery pressure differed between the two

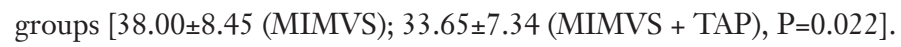

Conclusions: Our study showed that totally endoscopic mitral valve surgery with concomitant TAP is just as safe and effective as isolated totally endoscopic mitral valve surgery, even with a long surgery duration. Our study also suggested that totally endoscopic mitral valve surgery with concomitant TAP can improve tricuspid function in patients.

Keywords: Totally endoscopic; mitral valve surgery; tricuspid annuloplasty (TAP); tricuspid regurgitation; minimally invasive

Submitted Nov 14, 2020. Accepted for publication Mar 19, 2021.

doi: $10.21037 /$ jtd-20-3302

View this article at: http://dx.doi.org/10.21037/jtd-20-3302 


\section{Introduction}

Functional tricuspid regurgitation (FTR) is one of the most common concomitant diseases that presents in patients suffering from mitral valve disease, with a reported incidence of up to $64 \%$ (1-7). Recent studies have indicated that untreated FTR can develop in patients who only undergo isolated mitral valve surgery, and the progression of FTR can lead to high morbidity and mortality rates (8-10). Patients undergoing tricuspid annuloplasty (TAP) during mitral valve surgery can achieve an excellent clinical outcome without increased operative risk (11-13). Therefore, the latest European guidelines recommend concomitant TAP in patients undergoing mitral valve surgery, with mild to severe FTR and tricuspid annular dilation (14). However, most of the studies summarized above were conducted in the context of conventional open-heart surgery.

In the last two decades, with the introduction of minimally invasive techniques in the field of cardiac surgery, minimally invasive cardiac surgery has advanced quickly and been widely adopted in patients with mitral and tricuspid valve disease. However, previous guidelines have not paid much attention to minimally invasive cardiac surgery and did not provide a detailed explanation for minimally invasive mitral valve surgery (MIMVS) and concurrent tricuspid valve surgery.

Many people are concerned about complex manipulation, and prolonged cardiopulmonary bypass (CPB) time may be related to a higher occurrence rate of third-degree heart block and cerebral dysfunction. Therefore, retrospective research was conducted to evaluate the clinical outcomes of MIMVS with or without concomitant TAP in Fujian Union Hospital between January 2019 and June 2020. Propensity score matching (PSM) was performed to adjust for differences in baseline data and tricuspid valve parameters between the MIMVS-TAP and MIMVS groups. The main purpose of this study was to clarify the surgeryrelated parameters, surgical complications, and short-term outcomes and to analyse the clinical efficacy of concomitant TAP during totally endoscopic mitral valve surgery.

We present the following article in accordance with the STROBE reporting checklist (available at http://dx.doi. org/10.21037/jtd-20-3302).

\section{Methods}

\section{Patients}

From January 2019 to June 2020, a total of 143 consecutive patients who received totally endoscopic mitral valve surgery in our institution were enrolled. The study was conducted in accordance with the Declaration of Helsinki (as revised in 2013). This study protocol was ethically approved by the ethics committee of Fujian Medical University (2021QH008), and the requirement for written informed consent was waived because of its retrospective and anonymized nature. All enrolled patients were diagnosed with mitral valve disease and FTR, which can be treated under totally thoracoscopic guidance, and a minimally invasive approach was chosen by all the participants during the presurgical visit. The electronic medical records of all patients who had undergone MIMVS were reviewed. Included participants were required to meet the following criteria: (I) mitral valve disease treatment by thoracoscopic guidance; and (II) FTR. The exclusion criteria were as follows: (I) previous history of thoracic surgery; (II) severe peripheral vascular disease; (III) severe cardiac dysfunction (ejection fraction <35\%); (IV) chest wall deformities; (V) conversion to sternotomy incision; and (VI) coexisting severe aortic valve disease and/or coronary artery disease.

\section{Echocardiography}

Echocardiography was performed in all patients. Cardiac diastolic and systolic ventricular function and structure were assessed by transthoracic or transesophageal standard 2-D B-mode, M-mode, colour Doppler, and pulse and continuous Doppler echocardiography. The severity of valvular disease was assessed as recommended by the American Society of Echocardiography. The diameter of the tricuspid annulus was obtained in an apical 4-chamber view as the maximum distance between the base of the septal and posterior leaflet at the time of maximal valve opening. The severity of TR was assessed with an integrative approach as recommended by current guidelines, and qualitative and quantitative parameters, such as septal motion, the direction and size of the jet in colour Doppler, and the width of vena contracta, were considered collectively. The severity was categorized into four grades (grade $1=$ mild, grade 2 $=$ moderate, grade $3=$ moderate to severe, and grade $4=$ severe) (14-16).

Operative indications for secondary tricuspid regurgitation requiring mitral valve surgery were based on recommendations by experts and guidelines and were as follows: (I) severe TR; (II) significant tricuspid annular dilatation $\left(>40 \mathrm{~mm}\right.$ or $>21 \mathrm{~mm} / \mathrm{m}^{2}$ ) or signs of right heart failure; or (III) direct assessment during the valve 


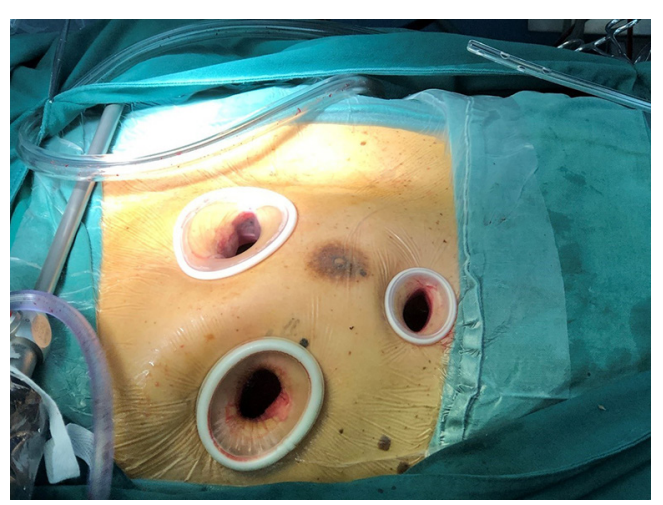

Figure 1 The surgical incisions for totally endoscopic cardiac surgery.

procedure, intraoperative saline testing, and comparison of the anterior and posterior leaflet surface area with the annulus size $(14,17)$.

The decision to perform TAP was dependent on the severity of TR, the diameter of the tricuspid annular, cardiac function, and the existence of atrial fibrillation, but the final decision was determined with the attending surgeon.

Short-term mortality or in-hospital mortality was defined as all-cause death within 30 days or any death that occurred during the hospital stay. Valve-related events were defined according to the guidelines published by Akins. Severe adverse events were defined as cerebrovascular accident, reintubation or tracheostomy, acute renal insufficiency, heart block, re-exploration surgery, bleeding and thromboembolic events, and sepsis (18).

\section{Surgical technique}

All procedures were performed using a totally endoscopic approach. All of our physicians who completed the learning curve were skilled at performing the totally endoscopic cardiac surgery. All surgical procedures were performed using standard steps. Patients in the two groups all received echocardiography prior to surgery to evaluate tricuspid regurgitation. After general anaesthesia and double-lumen intubation for deflating the right lung, patients were placed in a supine position with the right hemithorax slightly elevated.

A 3-5 $\mathrm{cm}$ oblique incision was made on the inguinal crease to obtain access to the femoral vessels. The CPB circuit was established by femoro-femoral cannulation with the guidance of transesophageal echocardiography. Venous drainage was provided by a single two-stage venous drainage cannula through the femoral vein to the superior vena cava. Systemic perfusion was achieved through the right femoral artery using the Seldinger technique (19).

The totally endoscopic approach was performed via an endoscopic right minithoracotomy (Figure 1). According to the method described by Ma et al., three chest wall ports were made for surgical manipulation (20). We used a transthoracic clamp to occlude the ascending aorta. Cardioplegic arrest was performed with antegrade HTK solution, and the right atrium was then opened. Under thoracoscopic direct vision, the femoral venous cannula was set in the correct position until unhindered access could be provided to the atrial septum (21).

First, mitral valve surgery was performed using the transseptal approach. After directly suturing the incision of the atrial septa, the tricuspid valve was evaluated by the surgical staff with saline testing or measuring the valve size with a sizer (Edwards MC3 Tricuspid Annuloplasty Ring, Edwards Lifesciences, USA). In cases of TAP, the tricuspid valve was repaired by suture annuloplasty, ring annuloplasty, or both, and surgical techniques were determined based on each surgeon's preference. After careful deairing of the heart, a transesophageal echocardiography evaluation of the heart was performed, CPB was disconnected, and all incisions were closed.

\section{Statistical analysis}

Statistical analysis was conducted using SPSS (IBM SPSS 24.0, SPSS Inc), and statistically significant differences were defined as $\mathrm{P}$-values $<0.05$. To minimize selection bias, PSM was performed to optimize the balance of baseline characteristics and tricuspid parameters. The propensity score for each patient was derived from a logistic regression model with adjustment for 10 key baseline variables, including age, sex, hypertension, body mass index, atrial fibrillation, New York Heart Association classification, left ventricular ejection fraction (LVEF), left ventricular end diastolic diameter (LVED), pulmonary artery systolic pressure (PASP), and the degree of tricuspid regurgitation. Nearest-neighbour 1:1 PSM without replacement with a calliper width of 0.05 was conducted. The propensity matching score was used to match 143 patients, and a total of 40 pairs ( 80 cases) were successfully matched.

All quantitative data with a normal distribution are expressed as the mean \pm one standard deviation. We used analysis of variance (ANOVA) or Student's $t$ test for comparisons of continuous variables, and the Mann-Whitney 
Table 1 Demographic and preoperative data comparing the two groups

\begin{tabular}{|c|c|c|c|c|c|c|}
\hline Item & \multicolumn{3}{|c|}{ Overall study patients } & \multicolumn{3}{|c|}{ Propensity-matched patients } \\
\hline Sex, M:F & $34: 58$ & 19:32 & 0.972 & $13: 27$ & $15: 25$ & 0.639 \\
\hline Age (years) & $57.84 \pm 10.37$ & $57.25 \pm 9.21$ & 0.739 & $56.78 \pm 10.84$ & $57.55 \pm 8.63$ & 0.724 \\
\hline BMI $\left(\mathrm{kg} / \mathrm{m}^{2}\right)$ & $22.67 \pm 1.44$ & $22.46 \pm 1.56$ & 0.405 & $22.65 \pm 1.38$ & $22.48 \pm 1.63$ & 0.63 \\
\hline Atrial fibrillation (n) & 38 & 21 & 0.832 & 15 & 19 & 0.366 \\
\hline NYHA [median, IQR] & 2 [2] & 2 [2] & 0.951 & 2 [2] & 2 [2] & 0.922 \\
\hline LVED (mm) & $58.60 \pm 8.20$ & $58.29 \pm 7.95$ & 0.831 & $57.58 \pm 8.25$ & $58.43 \pm 8.16$ & 0.644 \\
\hline LVEF (\%) & $56.71 \pm 6.15$ & $56.02 \pm 5.57$ & 0.511 & $56.80 \pm 6.01$ & $56.25 \pm 6.12$ & 0.684 \\
\hline Mitral stenosis & 30 & 20 & & 16 & 17 & \\
\hline Mitral insufficiency & 39 & 19 & & 15 & 14 & \\
\hline Mix lesion & 23 & 12 & & 9 & 9 & \\
\hline TR grade [median, IQR] & $2[1]$ & 3 [1] & $P<0.001$ & $3[1]$ & $3[1]$ & 1 \\
\hline
\end{tabular}

MIMVS, minimally invasive mitral valve surgery; TAP, tricuspid annuloplasty; NYHA, New York Heart Association classification; LVEF, left ventricular ejection fraction; LVED, left ventricular end diastolic diameter; PASP, pulmonary artery systolic pressure.

U-test was applied to compare nonnormally distributed variables. For categorical data, the chi-square test or Fisher's test was applied. Paired T-, Wilcoxon signed rank, and McNemar tests were applied in the PS matching cohort.

\section{Results}

One hundred and forty-three consecutive patients were selected for the study, 51 of whom underwent MIMVS with concomitant TAP (MIMVS-TAP group, N=51) and 92 of whom underwent isolated MIMVS (MIMVS group, $\mathrm{N}=92$ ).

\section{Comparison results of baseline data between the two groups before PSM}

There were no significant differences in terms of baseline data between the two groups. As shown in Table 1, there was a significant difference between the two groups regarding the severity of tricuspid regurgitation (detailed data before matching are shown in Table 1). If the two groups with different tricuspid valve-related parameters are compared, the comparative results will be uneven, and the persuasive conclusion will be weakened. Therefore, it is necessary to select patients with similar conditions for comparison between the two groups. In this case, PSM can achieve the statistical effect of the approximately random assignment of patients between the two groups.

\section{Comparison results of baseline data between the two groups after PSM}

The demographic data and tricuspid-related parameters were well matched between the two groups. The descriptive statistics for the study population are shown in Table 1. There were no significant differences in the baseline data, lesion type of mitral valve, or tricuspid regurgitation grade between the two groups.

\section{Comparison of perioperative outcomes between the two groups after PSM}

In the MIMVS + TAP group, a total of 22 patients underwent annuloplasty ring implantation, one patient underwent a Kay procedure, and 17 patients underwent the De Vega procedure. There were no significant differences in mitral valve surgery strategies. The concomitant maze 
Table 2 Intra-operative data compared between two groups after propensity score matching

\begin{tabular}{|c|c|c|c|}
\hline Item & MIMVS & MIMVS-TAP & $\mathrm{P}$ \\
\hline Mitral valve replacement & 27 & 27 & \\
\hline Mitral valve repair & 13 & 13 & \\
\hline Tricuspid valve surgery strategy (n) & & & 0 \\
\hline De Vega & 0 & 17 & \\
\hline Annuloplasty ring & 0 & 22 & \\
\hline Annuloplasty ring size [median, IQR] & $0[0]$ & $28[2]$ & $<0.001$ \\
\hline Maze procedure $(n)$ & 4 & 4 & 1 \\
\hline Aorta cross-clamp time (min) & $101.00 \pm 14.38$ & $110.80 \pm 17.37$ & 0.005 \\
\hline
\end{tabular}

MIMVS, minimally invasive mitral valve surgery; TAP, tricuspid annuloplasty.

procedure was also not significantly different. However, the total operation time $(204.25 \pm 28.49$ vs. $232.13 \pm 36.05 \mathrm{~min}$, $\mathrm{P}<0.001)$, CPB time $(153.10 \pm 23.00$ vs. $169.48 \pm 25.96 \mathrm{~min}$, $\mathrm{P}=0.004)$, and aorta cross-clamp time $(101.00 \pm 14.38$ vs. $110.80 \pm 17.37 \mathrm{~min}, \mathrm{P}=0.005)$ of the MIMVS + TAP group were significantly longer than those of the MIMVS group, as shown in Table 2.

The postoperative data are detailed in Table 3. Only one death among the patients was included in this study. One patient in the MIMVS group died of multiple organ failure. There were no significant differences observed in postoperative adverse events between the two groups.

There were no postoperative valve-related complications or embolization events in the MIMVS group. One patient in this group underwent re-exploration for bleeding. One patient suffered from postoperative acute renal failure and reintubation, and finally developed sepsis and died of multiorgan failure. Two patients had poor wound healing problems in chest incisions, four had subcutaneous emphysema, and four had pneumothorax in this group. Two patients had $\mathrm{AV}$ junctional rhythm requiring a temporary pacing wire.

One patient in the MIMVS-TAP group underwent reexploration surgery to stop bleeding from the incision site. A patient underwent endotracheal intubation again due to low blood oxygen saturation in the intensive care unit after the operation. During hospitalization, this patient developed acute renal insufficiency and was treated with haemodiafiltration, and eventually recovered and was discharged from the hospital. In the MIMVS-TAP group, one patient had lymphatic leakage in the inguinal incision, two patients had pneumothorax, and two patients had subcutaneous emphysema.

There were no differences in terms of the lengths of the intensive care unit stay and the postoperative hospital stay. In terms of cardiac structure and function, postoperative echocardiography showed no significant differences in left ventricular end-diastolic diameter or LVEF. However, the PASP $(38.00 \pm 8.45$ vs. $33.65 \pm 7.34 \mathrm{mmHg}, \mathrm{P}=0.022)$ and tricuspid regurgitation grade $(\mathrm{P}<0.001)$ were different between the two groups. No significant differences existed between the two groups for the incidence of conduction blocks and stroke.

\section{Discussion}

Many studies have indicated that left-sided surgery combined with TAP does not increase the operative risk in patients with FTR. Concomitant TAP can improve the functional status of the right ventricular $(4,11-13,22)$. According to the valvular heart disease guidelines set by the ESC/EACTS 2017, in patients suffering from left heart valve disease combined with TR, TAP was indicated for severe TR or mild-to-moderate TR with a dilated tricuspid 
Table 3 Postoperative data compared between the two groups after propensity score matching

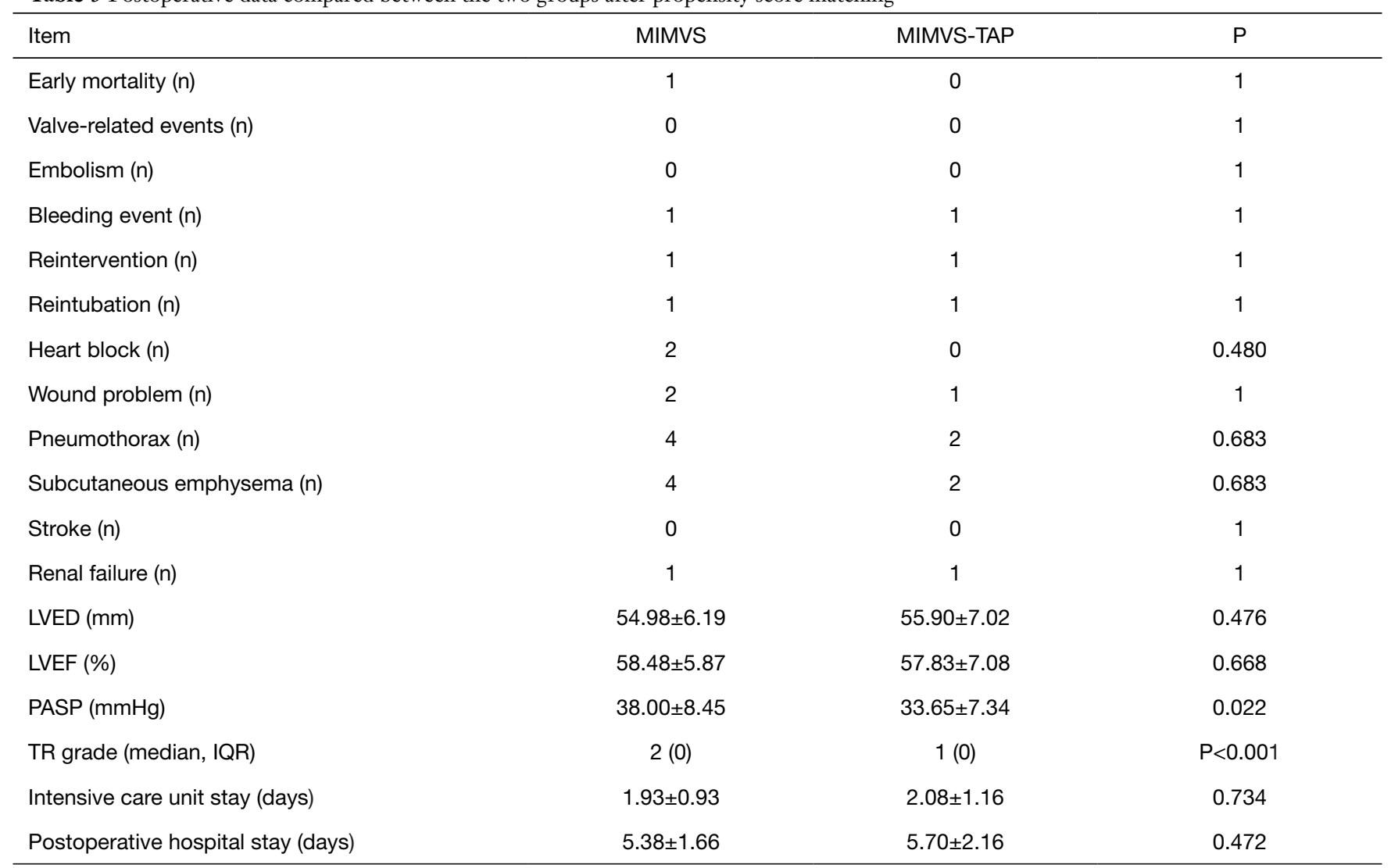

MIMVS, minimally invasive mitral valve surgery; TAP, tricuspid annuloplasty; LVEF, left ventricular ejection fraction; LVED, left ventricular end diastolic diameter; PASP, pulmonary artery systolic pressure.

annulus $\left(\geq 40 \mathrm{~mm}\right.$ or $\left.>21 \mathrm{~mm} / \mathrm{m}^{2}\right)$. The guidelines state that TAP can be beneficial for mild, moderate, or severe FTR during mitral valve surgery for patients with tricuspid annular dilatation (14).

However, the latest guidelines do not focus solely on totally endoscopic cardiac surgery. Since the 1990s, the introduction of thoracoscopy for cardiac surgery has been made possible with the development of minimally invasive and CPB techniques. Currently, the field of minimally invasive cardiac surgery is progressing rapidly and gradually expanding its indications. Several previous studies showed that there were no significant differences in the longterm and short-term clinical effects between the totally endoscopic approach and median sternotomy mitral valve surgery (23-26).

However, in most studies of totally endoscopic mitral valve surgery, concurrent tricuspid valve surgery has been completely ignored and not even discussed, despite the fact that tricuspid regurgitation has a high incidence rate. In contrast to routine median sternotomy and $\mathrm{CPB}$ procedures, the operating space is limited in minimally invasive cardiac surgery, which also requires a special perfusion strategy. The manipulations are complicated and require a longer operating time. In addition, there have been many concerns about the potential damage to the neurological system of patients who have undergone endoscopic cardiac surgery. The physical limitations of minimally invasive techniques, retrograde perfusion, and inadequate deairing may theoretically lead to a higher incidence of cerebral complications $(27,28)$.

According to the literature, FTR is commonly reported in mitral valve disease and must not be neglected. There is no doubt that endoscopic mitral and tricuspid valve surgery will increase the number of complicated surgical steps and the time of $\mathrm{CPB}$ procedures. Therefore, we conducted a study that focused on the clinical outcome of mitral valve surgery with or without TAP.

After examining the valve-related parameters, we found 
that there were notable differences in the severity of TR between the two groups. First, the proportion of TR was different. Thus, direct comparisons between the two groups cannot be conducted since the valve-related parameters were different, the perioperative results will be uneven, and the persuasive conclusion will be weakened. Therefore, we conducted PSM analysis, which can minimize selection bias. Second, as described earlier, the decision to perform tricuspid valve surgery is ultimately up to the surgeon, since the success of the procedure depends on the expertise and experience of the operators. Opinions about surgery can be very different. For severe FTR, most surgeons may prefer surgical treatment; for mild to moderate FTR, saline testing and measurements with the sizer are taken into consideration. Therefore, moderate and severe tricuspid regurgitation were also found in the isolated MIMVS group in this study, and comparisons were made after PSM between the two groups.

Our study demonstrated that patients undergoing MIMVS combined with TAP due to FTR did not have increased mortality and morbidity compared to patients receiving isolated MMIVS after PSM. TAP can be performed safely in patients undergoing MIMVS without increasing early mortality and morbidity rates or inducing conduction block and stroke.

There may be concerns that increased operative time and surgical steps of concomitant TAP in MIMVS may lead to an increased incidence of cerebrovascular accidents and three-degree atrioventricular block. Our study indicated that there were no differences in short-term mortality or adverse events and in fact this procedure led to a better clinical outcome with respect to the severity of tricuspid regurgitation and pulmonary artery pressure. We found that the degree of TR and PASP were reduced in the MIMVS-TAP group during the postoperative period. Previous studies have shown that concomitant TAP in patients undergoing mitral valve surgery can reverse right ventricular remodelling and improve the function of the right ventricle, especially in patients with a dilated tricuspid annulus (14,29).

Combined TAP with MIMVS prolonged the operative time. We found significant increases in aortic cross-clamp time and CPB time, which led to an increase in the total surgical time by nearly half an hour. The MIMVS+TAP procedure also cost significantly more, which was mainly related to the introduction of the ring prosthesis.
In general, our study found that mortality and morbidity rates, as well as stroke and conduction block rates, in MIMVS+TAP were not significantly different after matching compared with isolated MIMVS. In addition, it is obvious that MIMVS+TAP has great advantages over isolated MIMVS due to reduced TR progression along with decreases in right ventricular dysfunction $(4,22,29,30)$.

Although the clinical outcomes were satisfactory and demonstrated the efficacy of the surgery, our research study has some limitations. We still lack the long-term followup data to prove the long-term efficacy of MIMVS+TAP, to prove whether concomitant TAP in patients undergoing MIMVS can improve their long-term prognosis, and to determine whether concomitant TAP with MIMVS can reduce the progression of $\mathrm{TR}$ as a median sternotomy approach.

In addition, our research only introduced the totally endoscopic approach, which is only one of the minimally invasive approaches. Currently, for mitral valve surgery, there are several minimally invasive methods that are widely used such as the following: lower hemisternotomy, direct-vision right minithoracotomy, endoscopic right minithoracotomy, and robotic-assisted right minithoracotomy. The most suitable surgical approach should be compared among multiple centres in order to develop surgical selection strategies based on treatment effectiveness and economic factors. Furthermore, our cohort was small, and the followup period was short. Further follow-up is needed to collect data for long-term outcomes. However, despite these limitations, our findings still provide new evidence for the selection of surgical approaches in clinical practice.

\section{Conclusions}

Concomitant TAP in patients undergoing MIMVS is safe, even with a prolonged operative time. We found no difference in surgical mortality and morbidity after PSM. MIMVS combined with TAP can improve tricuspid function in patients.

\section{Acknowledgments}

We highly acknowledge the contribution by the participating doctors: Xue-Shan Huang, Feng Lin, Han-Fan Qiu, DongShan Liao and Qi-Min Wang.

Funding: None. 


\section{Footnote}

Reporting Checklist: The authors have completed the STROBE reporting checklist. Available at http://dx.doi. org/10.21037/jtd-20-3302

Data Sharing Statement: Available at http://dx.doi. org/10.21037/jtd-20-3302

Peer Review File: Available at http://dx.doi.org/10.21037/jtd20-3302

Conflicts of Interest: All authors have completed the ICMJE uniform disclosure form (available at http://dx.doi. org/10.21037/jtd-20-3302). The authors have no conflicts of interest to declare.

Ethical Statement: The authors are accountable for all aspects of the work in ensuring that questions related to the accuracy or integrity of any part of the work are appropriately investigated and resolved. The study was conducted in accordance with the Declaration of Helsinki (as revised in 2013). This study protocol was ethically approved by the ethics committee of Fujian Medical University (2021QH008), and the requirement for written informed consent was waived because of its retrospective and anonymized nature.

Open Access Statement: This is an Open Access article distributed in accordance with the Creative Commons Attribution-NonCommercial-NoDerivs 4.0 International License (CC BY-NC-ND 4.0), which permits the noncommercial replication and distribution of the article with the strict proviso that no changes or edits are made and the original work is properly cited (including links to both the formal publication through the relevant DOI and the license). See: https://creativecommons.org/licenses/by-nc-nd/4.0/.

\section{References}

1. Hung J. The pathogenesis of functional tricuspid regurgitation. Semin Thorac Cardiovasc Surg 2010;22:76-8.

2. Di Mauro M, Bezante GP, Di Baldassarre A, et al. Functional tricuspid regurgitation: an underestimated issue. Int J Cardiol 2013;168:707-15.

3. Calafiore AM, Iacò AL, Romeo A, et al. Echocardiographic-based treatment of functional tricuspid regurgitation. J Thorac Cardiovasc Surg 2011;142:308-13.

4. Dreyfus GD, Corbi PJ, Chan KM, et al. Secondary tricuspid regurgitation or dilatation: which should be the criteria for surgical repair? Ann Thorac Surg 2005;79:127-32.

5. McCarthy PM. Adjunctive procedures in degenerative mitral valve repair: tricuspid valve and atrial fibrillation surgery. Semin Thorac Cardiovasc Surg 2007;19:121-6.

6. De Bonis M, Lapenna E, Sorrentino F, et al. Evolution of tricuspid regurgitation after mitral valve repair for functional mitral regurgitation in dilated cardiomyopathy. Eur J Cardiothorac Surg 2008;33:600-6.

7. Shiran A, Sagie A. Tricuspid regurgitation in mitral valve disease incidence, prognostic implications, mechanism, and management. J Am Coll Cardiol 2009;53:401-8.

8. Izumi C, Iga K, Konishi T. Progression of isolated tricuspid regurgitation late after mitral valve surgery for rheumatic mitral valve disease. J Heart Valve Dis 2002;11:353-6.

9. King RM, Schaff HV, Danielson GK, et al. Surgery for tricuspid regurgitation late after mitral valve replacement. Circulation 1984;70:I193-97.

10. Song H, Kim MJ, Chung CH, et al. Factors associated with development of late significant tricuspid regurgitation after successful left-sided valve surgery. Heart 2009;95:931-6.

11. Bianchi G, Solinas M, Bevilacqua S, et al. Which patient undergoing mitral valve surgery should also have the tricuspid repair?. Interact Cardiovasc Thorac Surg 2009;9:1009-20.

12. Pfannmueller B, Verevkin A, Borger MA, et al. Role of tricuspid valve repair for moderate tricuspid regurgitation during minimally invasive mitral valve surgery. Thorac Cardiovasc Surg 2013;61:386-91.

13. Azarnoush K, Nadeemy AS, Pereira B, et al. Clinical outcomes of tricuspid valve repair accompanying left-sided heart disease. World J Cardiol 2017;9:787-93.

14. Baumgartner H, Falk V, Bax JJ, et al. 2017 ESC/EACTS Guidelines for the management of valvular heart disease. Eur Heart J 2017;38:2739-91.

15. Zoghbi WA, Enriquez-Sarano M, Foster E, et al. Recommendations for evaluation of the severity of native valvular regurgitation with two-dimensional and Doppler echocardiography. J Am Soc Echocardiogr 2003;16:777-802.

16. Lang RM, Bierig M, Devereux RB, et al. Recommendations for chamber quantification: a report from the American Society of Echocardiography's 
Guidelines and Standards Committee and the Chamber Quantification Writing Group, developed in conjunction with the European Association of Echocardiography, a branch of the European Society of Cardiology. J Am Soc Echocardiogr 2005;18:1440-63.

17. A. Carpentier, D.H. Adams, F. Filsoufi. Carpentier's Reconstructive Valve Surgery. Philadelphia: Saunders Elsevier, 2010:194-200.

18. Akins CW, Miller DC, Turina MI, et al. Guidelines for reporting mortality and morbidity after cardiac valve interventions. Ann Thorac Surg 2008;85:1490-5.

19. Bonaros N, Schachner T, Oehlinger A, et al. Robotically assisted totally endoscopic atrial septal defect repair: insights from operative times, learning curves, and clinical outcome. Ann Thorac Surg 2006;82:687-93.

20. Ma ZS, Dong MF, Yin QY, et al. Totally thoracoscopic repair of atrial septal defect without robotic assistance: a single-center experience. J Thorac Cardiovasc Surg 2011;141:1380-3.

21. Chitwood WR Jr, Elbeery JR, Moran JF. Minimally invasive mitral valve repair using transthoracic aortic occlusion. Ann Thorac Surg 1997;63:1477-9.

22. Van de Veire NR, Braun J, Delgado V, et al. Tricuspid annuloplasty prevents right ventricular dilatation and progression of tricuspid regurgitation in patients with tricuspid annular dilatation undergoing mitral valve repair. J Thorac Cardiovasc Surg 2011;141:1431-9.

23. Ritwick B, Chaudhuri K, Crouch G, et al. Minimally invasive mitral valve procedures: the current state. Minim
Invasive Surg 2013;2013:679276.

24. Galloway AC, Schwartz CF, Ribakove GH, et al. A decade of minimally invasive mitral repair: long-term outcomes. Ann Thorac Surg 2009;88:1180-4.

25. Modi P, Hassan A, Chitwood WR Jr. Minimally invasive mitral valve surgery: a systematic review and meta-analysis. Eur J Cardiothorac Surg 2008;34:943-52.

26. Stevens LM, Rodriguez E, Lehr EJ, et al. Impact of timing and surgical approach on outcomes after mitral valve regurgitation operations. Ann Thorac Surg 2012;93:1462-8.

27. Grossi EA, Loulmet DF, Schwartz CF, et al. Evolution of operative techniques and perfusion strategies for minimally invasive mitral valve repair. J Thorac Cardiovasc Surg 2012;143:S68-70.

28. Gammie JS, Zhao Y, Peterson ED, et al. Maxwell Chamberlain Memorial Paper for adult cardiac surgery. Less-invasive mitral valve operations: trends and outcomes from the Society of Thoracic Surgeons Adult Cardiac Surgery Database. Ann Thorac Surg 2010;90:1401-08.

29. Chikwe J, Itagaki S, Anyanwu A, et al. Impact of Concomitant Tricuspid Annuloplasty on Tricuspid Regurgitation, Right Ventricular Function, and Pulmonary Artery Hypertension After Repair of Mitral Valve Prolapse. J Am Coll Cardiol 2015;65:1931-8.

30. De Meester P, De Cock D, Van De Bruaene A, et al. Additional tricuspid annuloplasty in mitral valve surgery results in better clinical outcome. Heart 2015;101:720-6.
Cite this article as: Huang LC, Xu QC, Chen DZ, Dai XF, Chen LW. Hospital outcome of concomitant tricuspid annuloplasty during totally endoscopic mitral valve surgery: a propensity matched study. J Thorac Dis 2021;13(5):3042-3050. doi: $10.21037 /$ jtd-20-3302 